TRABAJOS DE INVESTIGACIÓN

\title{
Análisis de algunos factores socio-culturales en la enseñanza de un idioma extranjero
}

\author{
Analysis of some socio-cultural factors in teaching a foreign language
}

\author{
Pamela Tejeda Cerda, Angela Niebles Gutiérrez \\ Instituto de Ciencias de la Educación \\ Facultad de Filosofía y Humanidades \\ Universidad Austral de Chile \\ pamela.tejeda@gmail.com \\ aniebles@gmail.com \\ Telf.: (56) 63221262
}

\section{RESUMEN}

Este documento presenta una versión actualizada del artículo Análisis de algunos factores socio-culturales en la enseñanza de un idioma extranjero, escrito por el profesor Omar Silva en la primera edición de la Revista Estudio Pedagógicos de la Facultad de Filosofía y Humanidades de la Universidad Austral de Chile en el año 1976. La perspectiva de esta mirada es socio-crítica, proponiendo por un lado una retrospectiva de las propuestas originales, y por otro un análisis situado que permite desarrollar reflexiones globales en torno al fenómeno educativo y a la didáctica particular del aprendizaje de una segunda lengua.

Palabras clave: factores socioculturales, perspectiva socio-crítica, educación.

\begin{abstract}
This document is an updated version of the article "Analysis of some socio-cultural factors in teaching a foreign language" written by the teacher Omar Silva in the first edition of the Journal Estudios Pedagógicos from Facultad de Filosofía y Humanidades of Universidad Austral de Chile in 1976. The approach chosen is a socio-critical perspective which presents, on the one hand, a retrospective of the original proposals and, on the other, a located analysis which allows general considerations related to the educational phenomena and the specific didactics of learning a foreign language.

Key words: socio-cultural factors, socio-critical perspective, education.
\end{abstract}

El presente trabajo actualiza el contexto de lo que ha significado la enseñanza y aprendizaje del idioma extranjero, particularmente el inglés. Además, reflexiona sobre el actual sistema educacional en Chile, en donde pese a los cambios legislativos y políticos de las últimas décadas persisten los factores socioculturales enunciados por el autor original.

El aprendizaje de una segunda lengua, sigue principios didácticos inherentes al fenómeno del aprender, en este caso, nos interesa evidenciar la incidencia de los contextos de relación, en los resultados del aprendizaje. 


\section{ACTUALIZACIÓN RETROSPECTIVA DE LAS PROPUESTAS ORIGINALES DEL PROFESOR OMAR SILVA}

\subsection{SÍNTESIS DEL DOCUMENTO BASAL}

Silva (1976) realizó un estudio correlacional respecto a las variables: estrato socioeconómico y rendimiento académico de la asignatura inglés. Seleccionó una muestra de entre 600 y 700 estudiantes de enseñanza media (de un total de 10.000 aproximadamente), en la provincia de Valdivia.

Se aplicó un cuestionario sociocultural (datos personales y familiares) cuyos datos permitieron categorizar en estratos: alto, medio y bajo. Una vez delimitados, se emplearon para establecer una relación entre ellos y las notas obtenidas en cada nivel, las cuales se agruparon en dos categorías: estudiantes con nota superior a cuatro $(4,0)(\mathrm{S}-4)$ y estudiantes con nota inferior a cuatro $(4,0)(\mathrm{I}-4)$.

El análisis de los resultados se realizó mediante la décima X2 (Chi Square Test), la cual permitió concluir que existen diferencias significativas a nivel del $1 \%$ entre la variable socio-cultural y el rendimiento académico de la asignatura. El resultado permite afirmar que no existen antecedentes para pensar que el nivel o estrato sociocultural es independiente del rendimiento, es decir, es distinto en los diferentes niveles.

\subsection{FACTORES QUE INFLUYEN EN EL APRENDIZAJE DE UN SEGUNDO IDIOMA Y ORIENTACIONES PARA UN APRENDIZAJE EFECTIVO DEL INGLÉS}

El aprendizaje del inglés se entiende desde los resultados de los niveles de logros que establece la prueba SIMCE, centrados en las habilidades de reconocimiento y comprensión lectora y auditiva. Según señala la Agencia de Calidad de la Educación, los mayores resultados, por tanto del aprendizaje, están condicionados por los siguientes factores: estudiantes que comienzan clases de inglés antes del quinto año de enseñanza general básica, en donde la enseñanza es obligatoria; estudiantes con una distribución del inglés de al menos 4 horas a la semana, y un docente que realiza toda su clase en inglés. A partir de esta afirmación, para los estudiantes de sectores socioeconómicos bajos y pertenecientes a establecimientos municipales, el aprendizaje del idioma permanecerá en los niveles más bajos, reafirmando así el estudio correlacional realizado por Silva (1976).

Si bien el SIMCE se declara como una aplicación que busca evaluar y diagnosticar los niveles de aprendizajes del idioma, es importante señalar la doble lectura que tiene esta prueba. El riesgo de este sistema es la utilidad del inglés, el cual se está tornando cada vez más vinculante y, con ello, transformándose en un factor condicionante para los diferentes accesos sociales, culturales, de participación, entre otros, llegando a generar más brechas y desigualdades educativas y sociales de las ya existentes. Un claro ejemplo de ello es la Certificación de Nivel que entrega esta prueba para los resultados medio y avanzado (A2 y B1 respectivamente), ¿con qué fin una prueba estandarizada acredita niveles de aprendizaje mediante un certificado? ¿para qué es el uso o la emisión de un certificado en un nivel escolar? ¿en unos años más se solicitará el certificado para acceder a algún beneficio, oportunidad laboral y/o educativa?

Posicionando la mirada institucional sobre lo que en Chile, significa aprender inglés, el apartado siguiente presenta la evolución de las características básicas que, desde la perspec- 
tiva de Silva (1976), se constituyen en problemas inherentes a la situación de enseñanzaaprendizaje de un idioma extranjero, y que denominó factores socio culturales. Para efectos de este análisis, se han agrupado en tres componentes. El primero, sentidos y accesibilidad, incorpora los ítems a y b; relaciones sociales y económicas e incidencia en las oportunidades educativas, enfatiza el numeral c; y, apuntes didácticos, se compone de los puntos d y e.

El encuadre reflexivo que se desarrolla, está inspirado en Mey Yi Lin (2011) quien subraya "la importancia de entender la situación sociocultural, sociopolítica y socioeconómica del aprendizaje de una segunda lengua y de una lengua extranjera en diversos contextos del mundo" (p,12).

\subsubsection{Sentidos y accesibilidad}

a) La escasa utilidad de la asignatura de inglés al completar los alumnos la enseñanza media; b) La incapacidad de los mismos de utilizar el idioma en situaciones de comunicación lingüística después de seis o más años de estudios.

Pishgam (2011) señala cómo el acceso a diferentes bienes culturales, como internet, computadoras, imágenes, pinturas, libros, y la relación de los aprendices, con profesores, padres y hermanos y pares que manejen el idioma (capital social), puede tener una influencia en la forma en que un individuo pueda o no aprender una lengua.

Tras 40 años, el contexto ha cambiado. Lewis (2006) señala que actualmente la juventud tiene mayor manejo y acceso a internet, y que junto con la informática también aparece la telefonía celular o móvil, transformando los hábitos y costumbres de la comunicación. La facilidad y rapidez para contactar con otras personas que permiten las tecnologías de la información y comunicación (TIC) ha propiciado un aumento en la frecuencia de las comunicaciones. La aparición de redes sociales permite estar en contacto con personas que pueden vivir en otro país, tendencia que se da principalmente en jóvenes, creando comunidades virtuales respecto a un tema de interés. Este factor se transforma en un facilitador para la utilidad del inglés e incluso aporta a generar una actitud favorable de aprendizaje al idioma. Es decir, la gestión del conocimiento hoy por hoy logra, como afirma Carretero (2005), contribuir a un aprendizaje con perspectiva global, potenciando "el idioma inglés como medio de comunicación mundial y como herramienta imprescindible de acceso a fuentes de información" (2005: 3).

Una mayor utilidad del idioma inglés durante la enseñanza media permite que el (la) estudiante sea capaz de generar al menos una comunicación básica posterior al egreso de la enseñanza media, incluso después de seis o más años, según lo que propone el autor, considerando que en ese tiempo ya se encuentra realizando estudios superiores o bien desarrollándose en un ámbito laboral. Otros elementos a incorporar en esta reflexión, de acuerdo a Gholami (2012), citado en López, Quesada y Salas, son "las actitudes de los aprendices hacia su lengua meta, sus hablantes y el contexto" (2014: 7), ya que éstas son fundamentales en el logro y desarrollo de la competencia. La actitud y la motivación son necesarias cuando se trata de aprender otra lengua. Si los estudiantes no le encuentran "sentido", salvo el de la importancia, de aprender una segunda lengua, difícilmente tendrán una actitud positiva y disposición para el aprendizaje. La pregunta que subyace es el ¿para qué?, si no estoy en contacto con el idioma más que en el horario de clases. Diferente sería una propuesta de un conversatorio con estudiantes de un país de habla inglesa, su contexto es real y pueden evidenciar el "sentido" de comunicarse. 


\subsubsection{Relaciones sociales y económicas e incidencia en las oportunidades educativas}

c) El bajo rendimiento en la asignatura de inglés en aquellos establecimientos que atienden alumnos de niveles socioculturales medio y bajo, en oposición a aquellos de niveles socioculturales superiores.

La situación educativa chilena indica que los colegios privados gestionan y despliegan distintas posibilidades de acceso a contextos naturales donde se experimenta la segunda lengua, en comparación con aquellos particulares subvencionados, y más aún con los establecimientos de carácter público. Ramanathan (2006) invita a prestar atención a "la integración socioeconómica y sociopolítica de la enseñanza del inglés en una sociedad, especialmente la posición del inglés frente a otros idiomas en la sociedad, y cómo el acceso diferencial al inglés y a las pedagogías del inglés contribuyen a la estratificación social y a las inequidades" (citado en Mei Yi Lin, 2008: 16).

Chossudovsky (1980), consultor de la Comisión Económica para América Latina y el Caribe (CEPAL), afirma que la mirada fenoménica y los procedimientos empíricos son insuficientes para delinear la ruta estructural de la pobreza en los países de América Latina. Al parecer, la frontera de pobreza y el criterio sobre las necesidades humanas esenciales, promovidas por el Banco Mundial y el Fondo Monetario Internacional, ya son catalogadas como sesgadas desde esos años. El mismo autor explicita cómo las relaciones sociales y económicas inciden en las oportunidades educativas de diferentes grupos sociales. En tanto, la asignación del presupuesto educativo apoya implícitamente el mantenimiento de desigualdades sociales en cuanto a esas oportunidades.

Diferentes investigaciones en Latinoamérica confirman estas hipótesis. Cenoz y Perales (2000), y Moreno-Fernández (2007), examinan la influencia que las variables del contexto sociolingüístico y socioeducativo tienen en la adquisición de una segunda lengua (L2), indicando que el nivel socioeconómico también puede influir en la adquisición de la L2 en el medio escolar, lo que sigue ratificando la hipótesis inicial. Bosco (2008), en México; Villalta, Martinic y Guzmán (2011), en Chile; Sánchez y Otero (2012), en Colombia, encontraron hallazgos vinculantes entre las variables aprendizaje y nivel socioeconómico en variados sectores disciplinares y contextuales; Gelber, Treviño e Inostroza (2016) configuran las brechas de género de estudiantes de tercero y sexto grado en lectura, matemática, escritura y ciencias (sólo en sexto grado) en: Argentina, Brasil, Chile, Colombia, Costa Rica, República Dominicana, Ecuador, Guatemala, Honduras, México, Nicaragua, Panamá, Paraguay, Perú, Uruguay y el estado mexicano de Nuevo León, encontrando como una variable transversal las diferencias de desempeño según la estratificación social.

Como consecuencia del panorama ofrecido por la comunidad científica, podemos afirmar que esta desigualdad es el componente más determinante del grupo, propuesto inicialmente como factores invisibilizados que afectan el aprendizaje, en este caso de un segundo idioma. Desde el punto de vista pedagógico, se exhorta a abrir una ruta desde la perspectiva de Giroux (2004), quien plantea que, para una pedagogía radical que sea consciente de que existe una dialéctica entre estructura y participación, se requiere la utilización de los tres principios de organización de esta dialéctica: la reproducción, producción y reconstrucción (cambio intencionado), reconociendo entonces que la cotidianidad es siempre una posibilidad de agenciar incisiones que apelan a la homogenización y que pueden tener en cuenta el sentido del aprendizaje de una segunda lengua, logrando complejizar las motivaciones asociadas al exitismo y proponer nuevos 
horizontes, como el desarrollo comprensivo de las diversas culturas, tan como lo sugieren las didácticas más contemporáneas para el aprendizaje-enseñanza del inglés.

\subsubsection{Apuntes didácticos}

d) El uso de métodos tradicionales que no logran los cambios conductuales adecuados en los alumnos hacia el logro de una competencia lingüística también adecuada; e) Las condiciones pedagógicas en que se imparte la enseñanza: cursos muy numerosos, falta de medios audiovisuales y de contacto con el idioma, actitudes favorables o desfavorables determinadas socioculturalmente.

La hipótesis de partida afirma que "los profesores y lingüistas se han dado cuenta que en el desarrollo de la competencia comunicativa, el dominio de la mecánica de la lengua no asegura la habilidad para usar el idioma (repetición coral o individual, memorización de diálogos estructurados, etc.) en la comunicación; por el contrario, la práctica común y corriente retrasa la comunicación auténtica del idioma" (Savignon, 1972; citado por Silva, 1976: 20). Los estudios recientes, como el de Díaz-Larenas, Alarcón-Hernández, MartínezIlabaca, Roa-Ghiselini y Sanhueza-Jara (2015), investigan las variables didácticas del proceso de enseñanza y aprendizaje del inglés en Chile. Este equipo presenta tres modelos didácticos disciplinares que agrupan las directrices más sobresalientes en el área: el modelo gramática-producción, el modelo audiolingual y modelo comunicativo (MC), siendo este último el que prevalece en la actualidad.

Mei Yi Lin (2008) interpela la aplicación del MC, haciendo los siguientes aportes al método de aprendizaje-enseñanza, dimensionando su perspectiva socio-política:

- "Las metodologías de enseñanza del inglés y enseñanza comunicativa de lenguas han sido tratadas como una disciplina o tecnología neutral y objetiva que puede ser exportada de un país a otro" (2008: 15); este estilo didáctico no es neutral, involucra valores relacionales patriarcales que reproducen sus lógicas inherentes.

- La formación inicial docente de profesores de inglés debe tener en cuenta que "un curso estándar sobre currículo en enseñanza comunicativa de lenguas podría, con frecuencia, dejar mal preparados a estos profesores para enfrentar la resistencia estudiantil y las realidades del aula de clase, debido a la falta de atención a los deseos e identidades de los estudiantes" (2008: 18).

- Es necesario comprender y analizar el cómo los currículos, en muchos casos, "están diseñados a partir del deseo del Estado y de los empleadores por producir fuerza laboral capacitada en inglés en el futuro" (2008: 20).

Es claro, por un lado, que lo meramente lingüístico no basta para el desarrollo de la competencia lingüística, si ello no se inserta en un marco de realizaciones contextuales para la enseñanza del idioma extranjero, en este caso el inglés. Silva (1976) señala lo capital que resulta la propuesta de una metodología adaptable y flexible, dependiendo de los contextos socioculturales de los estudiantes (actitudes, motivaciones, cultura, intereses), sin duda antecedentes previos que todo profesor o profesora de idiomas deben considerar para la planificación y ejecución de la enseñanza.

Autores como Navarro (2010) y Matanzas (2016) confirman tales planteamientos desde la base de la interacción social y la motivación integradora, respectivamente, posicionándose de manera más clara el enfoque sociolingüista como alternativa pedagógica. 
Es fundamental visibilizar que la didáctica disciplinar del inglés tiene una especial responsabilidad que supera la adquisición de una competencia lingüística, comprendiendo que el aprendizaje de una segunda lengua es una comprensión socio-cultural de un contexto ajeno al local, que trae, por tanto, improntas estructurales asociadas a visiones políticas, económicas y valóricas que deben ser decodificadas y transparentadas, contrastando también la riqueza de los universos interculturales del propio país o región.

Por otro lado, incorporar el inglés a temprana edad ha sido en el último tiempo la estrategia para mejorar los niveles de aprendizaje en cursos posteriores, entendiendo que los niños y niñas son más eficientes y efectivos en el aprendizaje de una segunda lengua que los adultos y adolescentes (Sadeghi, 2013).

\section{ANÁLISIS SOCIO-POLÍTICO EN TORNO AL FENÓMENO EDUCATIVO Y A LA DIDÁCTICA PARTICULAR DEL APRENDIZAJE DE UNA SEGUNDA LENGUA}

\subsection{LA OBLIGATORIEDAD DEL INGLÉS COMO SEGUNDO IDIOMA EN CHILE}

El siguiente recorrido histórico ejemplifica la relevancia del inglés como segunda lengua para nuestro país, y con ello un subsector de aprendizaje, que tras el Sistema de Medición de la Calidad de la Educación (SIMCE), posiciona con niveles de logro tanto a sus estudiantes como al establecimiento.

En Chile, desde 1980 se establece la obligatoriedad para la enseñanza de una segunda lengua, incorporando así dos idiomas extranjeros para $7^{\circ}$ y $8^{\circ}$ grado de enseñanza básica. Generalmente una de ellas fue la lengua inglesa y la segunda estaba sujeta a la orientación del establecimiento (francés, italiano y/o alemán).

Tras la reforma educativa en el año 1999, se propuso un solo idioma extranjero obligatorio desde $5^{\circ}$ grado de educación básica hasta primer año de enseñanza media. Si bien no se especificó cuál idioma debería impartirse, se asumió de manera espontánea la lengua inglesa.

En el año 2003, el Ministerio de Educación (MINEDUC) creó el Programa Inglés Abre Puertas (PIAP), para fomentar la enseñanza y el aprendizaje del inglés en el país, mejorando los niveles del idioma para estudiantes de $8^{\circ}$ grado de enseñanza básica y $4^{\circ}$ año de enseñanza media, como también para los y las docentes de inglés. Del programa se desprende la Red de Docentes de Inglés (RDIs) como un espacio de reflexión y colaboración entre pares, con la finalidad de adaptarse a las nuevas demandas que plantea la enseñanza y aprendizaje del inglés en el sistema escolar chileno.

En el año 2010, se aplica por primera vez la prueba SIMCE en el subsector de inglés, elaborada por el Cambridge English Language Assessment, para estudiantes de tercer año de enseñanza media. Esta prueba arrojó como resultado que los niveles de logro son bajos, tanto en comprensión lectora como auditiva. Los niveles de desempeño altos favorecen a los estudiantes de sexo masculino, pertenecientes a establecimientos científico-humanista, particular-pagado y de nivel socioeconómico alto. Los estudiantes provenientes de los sectores más vulnerables son los que muestran resultados más bajos en los aprendizajes evaluados (Agencia de Calidad de la Educación, 2010).

Los resultados del SIMCE 2014 aplicado al tercer año de enseñanza media en la Región de Los Ríos arrojó datos preocupantes. Los puntajes obtenidos, tanto en las 
secciones comprensión lectora como comprensión auditiva, establecieron un total de 44 puntos significativamente más bajo respecto al promedio nacional de 50 puntos. Asimismo, los resultados nacionales según niveles $\mathrm{CERF}^{1}$ apuntan a que un $67,6 \%$ se encuentra bajo el nivel A1, siendo éste el porcentaje más alto a nivel nacional. Esto quiere decir que el estudiante no logra los niveles de aprendizaje para: comprender expresiones familiares comunes y frases muy básicas con el objeto de satisfacer necesidades específicas; identificar el sentido del material escrito sencillo con descripciones simples, especialmente si están acompañadas de apoyo visual en imágenes; y reconocer nombres, palabras y frases muy básicas que se encuentran en letreros y en situaciones cotidianas (Agencia de Calidad de la Educación, 2014).

Thieme (2010) afirma que las reformas educativas iniciadas en Chile en la década de los 80 han fracasado, ya que no ha disminuido la brecha entre los estudiantes de distintos grupos socioeconómicos y tampoco se han incrementado los niveles de desempeño en pruebas internacionales. La inequidad sigue siendo un factor permanente y que no parece tener vuelta en la praxis, pues los discursos asociados a la calidad y la necesidad de la equidad y la igualdad pululan como una moda, pero en la cotidianidad la diferencia entre los establecimientos privados, subvencionados y estatales se mantiene.

\section{REFLEXIONES E IMPLICACIONES}

A lo largo del texto se evidencia cómo el desarrollo de la competencia sociolingüística en el proceso de adquisición de una segunda lengua está ligada a diversos contextos o sistemas de relación que permiten sostener que, pese a los cambios legislativos y políticos en el tema, permanecen elementos discriminatorios que sostienen la brecha de desigualdades y oportunidades en el sistema educacional chileno desde hace 40 años.

La educación y el desarrollo de didácticas inclusivas e integradoras avanzan de manera lenta de cara al ritmo global. Se observa que las especificidades a nivel de la didáctica disciplinar no han variado significativamente, se sigue viendo el aprendizaje como una sumatoria de factores intrínsecos y extrínsecos, que danzan como ítems y no como una totalidad corpórea interactuante. Si se logra posicionar el aprendizaje como proceso social y se sitúa el uso social de la lengua, se induce la incorporación de factores discursivos y situacionales propios de la sociolingüística ${ }^{2}$.

Las nociones planteadas por Bauman, respecto al homo economicus y el homo consumens como constructos definitorios de la sociedad de mercado, se ven reflejadas de manera implícita en las apuestas políticas neoliberales que se afianzan en la legislación educativa chilena, se reflejan en 1999, cuando se inserta la obligatoriedad y posicionamiento

El CERF (Common European Framework of Reference for Languages) establece seis niveles comunes de referencia para el aprendizaje de un idioma: A1, A2, B1, B2, C1, C2, los que representan tres amplios niveles: Usuario básico (A), Usuario Independiente (B) y Usuario Competente (C). Los niveles están relacionados con el Marco común europeo de referencia para las lenguas: enseñanza, aprendizaje, evaluación.

2 "Los sociolingüistas, tienen en cuenta que los aprendices están condicionados por factores sociales, como su extracción socioeconómica, su etnia o incluso su sexo/género, que la acción de los hablantes -también la de los aprendices- se realiza por medio de actos comunicativos, que les permiten tanto transmitir significados como relacionarse con sus interlocutores, y que la acción comunicativa es una actividad -no producto- que emerge en cada contexto con la propia práctica lingüística" (Moreno-Fernandez, 2007: 58). 
de la lengua inglesa como segundo idioma, popularizando la necesidad de dominar el inglés como promesa de progreso y de éxito. La posibilidad de alcanzar este hito, es accesible de forma más expedita para los estratos socio-económicos que logran estudiar en establecimientos privados.

\section{REFERENCIAS BIBLIOGRÁFICAS}

Agencia de la Calidad de la Educación (2014). Obtenido de http://archivos.agenciaeducacion.cl/ resultados-2014/Sintesis_Resultados_IIIM_2014.pdf

Agencia de la Calidad de la Educación (2010). Obtenido de http://www.agenciaeducacion.cl/wpcontent/files_mf/informenacionalderesultadossimce2010247mb.pdf

Bosco, E. B. (2008). Factores escolares asociados a los aprendizajes en la educación primaria mexicana: un análisis multinivel. REICE: Revista Electrónica Iberoamericana sobre Calidad, Eficacia y Cambio en Educación, vol. 6 (1), 58-84.

Cenoz, J., y Perales, J. (2000). Las variables contextuales y el efecto de la instrucción en la adquisición de segundas lenguas. Segundas lenguas. Adquisición en el aula, 109-125.

Chossudovsky (1980). Metodología y análisis de la pobreza en América Latina. Santiago: Comisión Económica para América Latina y el Caribe. (CEPAL).

Díaz-Larenas, C., Alarcón-Hernández, P., Martínez-Ilabaca, P., Roa-Ghiselini, I., y Sanhueza-Jara, M. G. (2015). Docentes de inglés de primaria, secundaria y terciaria: Sus creencias pedagógicas sobre sus estudiantes. Revista Educación, vol. 39 (2), 43-62.

Gelber, D., Treviño, E., e Inostroza, P. (2016). Inequidad de género en los logros de aprendizaje en educación primaria ¿Qué nos puede decir TERCE?: resumen ejecutivo. Santiago: UNESCO.

Giroux, H. (2004). Teoría y resistencia en educación: una pedagogía para la oposición. México: Siglo XXI.

Levis, D. (2006). El chat: El habla escrita de niños y jóvenes. Ponencia presentada en ALAIC.http:// archivos.agenciaeducacion.cl/resultados2014/Sintesis_Resultados_IIIM_2014.pdf

López, R. L., Quesada, M. J. Q., y Salas , J. S. (2014). Social Factors Involved in Second Language Learning: A Case Study from the Pacific Campus, Universidad de Costa Rica. Revista de Lenguas Modernas, 20, 435-451..

Matanzas Rodríguez, M. D. L. Á. (2016). La motivación en la adquisición de una segunda lengua. Repositorio abierto Universidad de Cantabria. Recuperado de http://hdl.handle.net/10902/8829

Mei Yi Lin , A. (2008). Cambios de paradigma en la enseñanza de inglés como lengua extranjera: El cambio crítico y más allá. Revista de educación y pedagogía, vol. 20 (51), 11-23.

Ministerio de Educación de Chile (2013). Resultados SIMCE III Medio 2012. Recuperado el 13 de septiembre 2016 en http://www.mineduc.cl/usuarios/mineduc/doc/201306061729100. Resultados_SIMCE_ingles_III_Medio_2012.pdf

Moreno Fernández, F. (2007). Adquisición de segundas lenguas y Sociolingüística. Revista

de Educación, 343, 55-70.

Navarro Romero, B. (2010). Adquisición de la primera y segunda lengua en aprendientes en edad infantil y adulta. Revista de iniciación a la investigación en filología, vol. 2, 115-128. Consultado en http://www.ual.es/revistas/PhilUr/pdf/PhilUr2.2010.Navarro.pdf

Carretero Ramos, A. (2005). Las TICS en el aula de Inglés: un proyecto de trabajo. Recuperado de: http://www.quadernsdigitals.net/index.php?accionMenu=hemeroteca.VisualizaArticuloIU. visualiza\&articulo_id $=8656$

Pishghadam, R., Noghani, M., y Zabihi, R. (2011). An application of a questionnaire of social and cultural capital to English language learning. English Language Teaching, vol. 4 (3), 195-203.

Sadeghi, K. (2013). Learner's Starting Age of Learning EFL and Use of Language Learning 
Strategies. Published by Canadian Center of Science and Education, Vol. 6, № 1. Recuperado desde www.ccsenet.org/elt

Sánchez, A. y Otero, A. (2012). Educación y reproducción de la desigualdad en Colombia. Reportes del emisor, 154, 1-4.

Thieme, C. (2010). Elección de establecimientos Educacionales en Chile ¿Por qué no ha existido presión por Calidad?. Santiago: Universidad Diego Portales. Documentos de Trabajo, 2.

Villalta Páucar, M. A., Martinic Valencia, S., y Guzmán Droguett, M. A. (2011). Elementos de la interacción didáctica en la sala de clase que contribuyen al aprendizaje en contexto social vulnerable. Revista mexicana de investigación educativa, vol. 16 (51), 1137-1158. 
